\title{
Irrelevant thoughts, emotional mood states, and cognitive task performance
}

\author{
PENNIE S. SEIBERT \\ Boise State University, Boise, Idaho \\ and \\ HENRY C. ELLIS \\ University of New Mexico, Albuquerque, New Mexico
}

\begin{abstract}
In two experiments, we investigated the relationship shared by irrelevant thoughts, emotional mood states, and cognitive task performance. At an empirical level, irrelevant thoughts were defined as thoughts that did not facilitate successful task performance. We used the same general procedure for both experiments: three groups of college students received happy-, neutral- (control), or sad-mood inductions and performed a memory task. The procedure for obtaining thoughts varied between experiments. The subjects in Experiment 1 listed their thoughts after the memory recall task. In Experiment 2, the subjects were tape-recorded while performing a memory task and producing concurrent verbal protocols. The subjects in both experiments then judged their thoughts in terms of frequency, intensity, and irrelevance. We found a similar pattern of results in both experiments: (1) The proportions of irrelevant thoughts and recall performance were negatively related, and (2) happy and sad students produced reliably greater proportions of irrelevant thoughts than did neutral (control) students.
\end{abstract}

Every day, people experience emotional mood changes that influence performance. Yet the underlying dynamics of these effects are not clear. Emotional states have been shown to influence cognitive processes in a variety of ways (Alloy \& Abramson, 1979; Anooshian \& Siegel, 1985; Bower \& Mayer, 1989; Clark, Milberg, \& Erber, 1988; Ellis \& Ashbrook, 1989; Ellis, Seibert, \& Herbert, 1990; Forgas \& Bower, 1988; Isen, 1984; Kenealy, 1986; Ucros, 1989). These investigations have shown that emotional mood states are related to (1) alterations in social and personal judgments, (2) alterations in spatial judgments, (3) mood congruence effects, and (4) recall impairment. The latter effect, recall impairment, will be the focus of the present investigation. Impairments in recall performance are observed when a complex task occurs in the context of relatively intense emotional mood states (Ellis, 1985; Hasher \& Zacks, 1979).

One way to study the effects of emotional states is to study thoughts, because thoughts vary in harmony with emotional mood states. Thoughts associated with emotional states can inspire or impair performance. These thoughts are often self-referent in nature. For example, in their research on test anxiety, Sarason and his col-

This research was conducted at the University of New Mexico under the direction of Henry C. Ellis in partial fulfillment of the requirements for Pennie Seibert's doctoral degree. The authors wish to thank Peder Johnson, University of New Mexico, Linda Anooshian, Boise State University, and Robert Proctor, Purdue University, for their review and comments on this research. Correspondence should be addressed to Pennie S. Seibert, Department of Psychology, 1910 University Drive, Boise State University, Boise, ID 83725. leagues (Sarason, 1984; Sarason, Sarason, Keefe, Hayes, \& Shearin, 1986; Sarason \& Stoops, 1978) found that subjects who were anxious about tests experienced cognitive interference that was peculiar to emotional states associated with test anxiety. These anxious states included thoughts pertaining to increased social anxiety or worry, increased intensity of fear of failure, and increased negative self-evaluation. These studies showed that while taking exams, individuals who were anxious about tests reported greater numbers of thoughts related to worrying about their performance than did nonanxious individuals. Thus, it appears that anxious people engage in thoughts that are qualitatively and quantitatively different from those of nonanxious people, and that these thoughts are related to performance.

In addition, Ingram, Kendall, Smith, Donnell, and Ronan (1987) demonstrated that both depressed and anxious individuals show patterns of selective information processing. Depressed people selectively processed depressing information, and anxious people selectively processed anxiety-causing information. The depressed engaged in more depressive thinking and the anxious engaged in more anxious thinking, both of which led to patterns of dysfunctional attributions and cognitive interference. The reported dysfunctional attributions included thoughts in which the subjects overemphasized possible negative outcomes; cognitive interference was associated with negative thoughts and contributed to poor performance on a wide range of tasks. Similarly, Beck's model of depression (Ottaviani \& Beck, 1988) postulates a negative cognitive triad, suggesting that clinically depressed people view themselves, their worlds, and their futures in a negative 
way. These studies suggested that the type of thoughts produced influence and interfere with other thoughts and behaviors.

Most of the literature concerning cognitive impairment, as reviewed above, has focused on anxious or depressed emotional states. Yet, it is reasonable to hypothesize that any emotional state includes emotion-specific thoughts, regardless of whether it is a positive or a negative emotional state. Hence, any emotional state could include extraneous or task-irrelevant thoughts that could result in cognitive impairment. The old adage that happy moods promote successful performance may not be the case, particularly if the criterion task is difficult and the happy emotional mood state is sufficiently intense. We investigated thoughts related to happy and sad states to find out whether both positive and negative states impair cognitive performance and to find out whether both states are related to increased productions of irrelevant thoughts.

Whether happy or sad, any thoughts that take the focus away from thoughts appropriate to and necessary for criterion task performance could interfere with performance. We consider these attention-diverting thoughts to be $i r$ relevant thoughts, because they are irrelevant to the successful solution of the criterion task. Accordingly, for the present research, we empirically defined irrelevant thoughts as thoughts that did not facilitate successful solution of the criterion task. Our irrelevant-thought category included thoughts about internal states such as those relating to the experience of an emotional state, about irrelevant features of a criterion task, and about any other distractions. Conversely, thoughts used to facilitate and focus on successful task performance were defined as relevant.

By definition, irrelevant thoughts are distracting; they interfere with successful task performance because attention is diverted, perhaps leaving fewer resources to perform the criterion task. This definitional base predicts that increases in irrelevant thoughts will be associated with decreases in successful task performance, regardless of whether relatively intense emotional mood states are experienced. This prediction is consistent with models of attention (e.g., Kahneman, 1973). A logical extension of this prediction assumes that if irrelevant thoughts increase during emotional mood states, successful cognitive performance will be at greater risk in emotional contexts (Ellis \& Ashbrook, 1988; Seibert \& Ellis, 1991b).

Researchers have employed various thought-listing procedures to assess thoughts (Ellis, Seibert, \& Herbert, 1990; Folkman \& Lazarus, 1985; Heimberg, Nyman, \& O’Brien, 1987; Seibert \& Ellis, 1991b). For example, Seibert and Ellis (1991b) asked subjects who were in happy, neutral, or sad mood states to list all the thoughts that came to mind while they engaged in a memory task. After having listed the thoughts, the subjects identified the thoughts that facilitated successful task solution and those that did not. Using a delayed thought-listing procedure, Seibert and Ellis obtained a simple measure of irrelevant thoughts and a listing of the types of thoughts that occurred. They found that both happy and sad in- dividuals produced more irrelevant thoughts than did neutral individuals (controls). We developed and refined their procedure by using convergent thought-listing procedures and a more sensitive measure of irrelevant thoughts that incorporated ratings of intensity and frequency of the thoughts.

We used a delayed thought-listing procedure in Experiment 1 , which required the subjects to write a list of their thoughts after task performance. This procedure allowed the subjects to perform the task with as little interference as possible. The subjects were unaware that they would later be instructed to report thoughts that occurred during task performance. However, a potential confounding may have entered when the subjects listed thoughts after the recall task. At this point, the subjects may have been aware of their level of performance on the recall task. This knowledge could have influenced not only the thoughts listed but also their rating of the thoughts. It was possible that the subjects who performed poorly could have reported more irrelevant thoughts to make an excuse for their performance.

Another potential issue with regard to the delayed thought-listing procedure concerns whether all thoughts were sampled equally. In other words, if thought-listing occurs after the task, only certain thoughts may be available. For example, Folkman and Lazarus (1985) found that different types of thoughts occurred during different stages of an examination. If different types of thoughts occur during different stages of a task, thoughts should be sampled throughout the task.

We used a concurrent verbal protocol thought-listing procedure in Experiment 2 to address the concerns noted above. This procedure introduces a set of possible confoundings that does not apply to the delayed procedure used in Experiment 1. When subjects are required to "think aloud," the criterion task becomes more difficult because the cognitive load increases. Also, interference and irrelevant thoughts (thoughts about the tape recorder) are created by the presence of the tape recorder. Subjects are aware that their thoughts are tape-recorded and attend to that fact. Still, the concurrent verbal protocol procedure rendered the converging operation we needed.

In summary, our objective was to examine the relationship among irrelevant thoughts, cognitive task performance, and emotional mood states. We hypothesized that (1) irrelevant thoughts increase in the context of emotional mood states, and (2) irrelevant thoughts and successful cognitive task performance are negatively related, regardless of emotional mood state. We used two different thought-listing procedures to test these hypotheses; the two experiments will be reported together, because of their methodological similarity.

\section{METHOD}

\section{Subjects}

We tested a total of 90 introductory psychology students at the University of New Mexico, 45 per experiment, and we randomly 
assigned 15 subjects to each of three mood conditions (happy, neutral, and sad) in each experiment

\section{Design}

Prescreening. The Beck Depression Inventory (BDI; Beck, 1978) was used as a prescreening instrument (1) to ensure that subjects who came to the experiment in a depressed state would not participate in mood induction (students who scored over 13 were not included in the study), and (2) to provide a measure of the comparability of groups prevailing mood states prior to mood induction.

Mood induction and assessment. We used the Seibert and Ellis (1991a) mood-induction procedure to induce happy, neutral, and sad moods. This procedure includes 25 self-referent statements presented at 20-sec intervals. A sample happy statement is. "Life's a blast, I can't remember when I've felt so good." A sample sad statement is, "I feel I am being suffocated by the weight of my past mistakes." And finally, an example of a neutral statement is, "There are $60 \mathrm{sec}$ in one minute." The items in the sad and hoppy conditions are ordered to become progressively more intense in their mood effects.

Mood induction was assessed with the Depression Adjective Checklist (DACL; Lubin, 1965), which provides a continuum for the measurement of mood states. It was designed to assess moods that are more temporary in nature, rather than more enduring moods. The subjects were instructed to check the adjectives from the list that were most descriptive of their feelings at the time ("how you feel now"). This measure includes 21 sad-mood adjectives (e.g., distressed, unhappy) and 11 happy-mood adjectives (e.g., peaceful, good). To score the subject, one sums the number of sad-mond words checked and the number of happy-mood words not checked. Higher scores indicate more sad-mood states.

Memory task. A perceptual grouping task was used that provided the opportunity for subjects to either recall the sequence as presented (i.e., BO NKI D, which is not very meaningful or pronounceable) or to reorganize the sequence into a more meaningful structure (such as BON KID, which is more meaningful and consists of two pronounceable units). The perceptual grouping memory task has been used in several studies (e.g., Bower \& Winzenz, 1969: Ellis. Parente, \& Walker, 1974; Seibert \& Ellis, 1991b) and is relatively demanding (Leight \& Ellis, 1981). This task was selected because the effects of experimentally induced mood states on memory are likely to be greatest with relatively difficult, demanding tasks (Ellis. 1985; Ellis \& Ashbrook, 1988).

\section{Procedure}

Seven trigram doublets were grouped as follows: R ITW EL, LI NF UZ, BO NKI D, NA TV IM, W ISHI T, B AHLI T, and CA MRE P; they were presented on $3 \times 5$ in. index cards. The subjects were shown all letter strings for one presentation of $2 \mathrm{~min}$, and they were instructed to study the sequences for later recall. No instructions or suggestions were given about regrouping the sequences; thus, subjects were free to study and encode the letter strings as they wished. Recall was accomplished by allowing $3 \mathrm{~min}$ for the subjects to write all of the letters remembered on a sheet of paper printed with seven lines of six equally spaced dashes until all of the dashed lines were filled, creating a total of 42 letters (i.e., 42 possible correct responses; a correct score consisted of a correct single letter). After $2 \mathrm{~min}$, the subjects were told to guess the remaining letters so that all of the dashed spaces were filled.

Thought-listing. In Experiment 1, immediately after the recall task, the subjects were given a sheet of paper upon which 10 rectangular boxes were printed, and they were instructed to list all of the thoughts that came to mind during the perceptual grouping task. The subjects were instructed to write one thought per box, and they were told that complete sentences were not needed. If the subjects completed all 10 boxes before the alloted $2.5 \mathrm{~min}$ had expired, they were given another sheet of paper with 10 more boxes to list further thoughts.
In Experiment 2, the subjects were informed that the purpose was to obtain a listing of all the thoughts that occurred while they performed a task during an emotional mood state. The subjects were told that all of their thoughts were important, regardless of whether the thoughts pertained to how to perform the task or not. The experimenters emphasized that the subjects were to express all thoughts that came to mind during task periormance-regardless of the thought content. The resulting verbal protocols were tape-recorded. If a subject stopped verbalizing thoughts at some point during the experiment, the experimenter encouraged the subject to "think aloud."

Thought-rating. After the thought-listing was accomplished, the subjects were instructed to rate their thoughts in three ways. First, they rated their thoughts according to whether they felt the thought helped or hindered their performance of the task (that is, taskrelevant and -facilitative or task-irrelevant and -nonfacilitative). Second, the subjects reported the judged frequency of that thought's occurrence. Third, the subjects rated the judged intensity of each thought by indicating whether it had a strong, somewhat strong. somewhat weak, or weak effect on their successful performance of the task.

We used the subjects' identification of their irrelevant thoughts, because pilot work done to compare designations of relevant versus itrelevant thoughts by subjects who listed the particular thoughts with designations made by experimenters revealed that the subjects' designations were more accurate. The subjects were better able to identify thoughts that had personal relevance for successful task solution than were the experimenters.

In addition to judging their thoughts for relevance or irrelevance, the subjects rated the intensiry and frequency of their thoughts. Subjects may experience a paricular thought more than once, yet will list it only once: also, the listed thoughts vary in intensity. To increase the sensitivity of the measure of the listed thoughts, the subjects were asked to estimate how often they experienced each thought and to rate the intensity of the thought according to its effect (from 4 = strong effect to 1 = weak effect) on successful performance of the memory task.

Immediately after the subjects' consent was obtained, the BDI was administered; subjects who scored 13 or under were informed of the nature of mood induction, and subjects who scored over 13 were released. The next step was mood induction, according to one of the three randomly assigned mood conditions (happy, neutral, or sad), followed by the DACL. After this, the subjects participated in the memory task, which was followed immediately by thought-listing in Experiment 1. Concurrent thought-listing occurred during the recall task in Experiment 2. Thought-listing was followed by thought-rating procedures. The subjects who received either sador neutral-mood inductions received 10 happy items just prior to debriefing. The entire procedure took less than $1 \mathrm{~h}$.

\section{RESULTS}

\section{Comparability of Groups}

The average BDl score was assessed for each group. In Experiment 1 (delayed thought-listing), mean scores for the happy, neutral, and sad groups were 4.67, 5.87, and 5.95, respectively $[F(2,42)=.72, p>.05]$. In Experiment 2 (concurrent protocols), mean scores for the happy, neutral, and sad groups were $6.67,4.53$, and 5.13 , respectively $[F(2,42)=1.43, p>.05]$. The mood induction groups' prevailing mood states were not reliably different prior to the induction procedure.

\section{Assessment of Mood-Induction Procedure}

Reliable mood induction effects were obtained in both experiments. In Experiment 1, the mean scores for the 
happy, neutral, and sad groups were $3.73,6.87$, and 16.40 , respectively $[F(2,42)=45.68, p<.001]$. In Experiment 2 , the mean scores for the happy, neutral, and sad groups were $4.80,8.13$, and 14.40 , respectively $[F(2,42)=15.83, p<.001]$. For both experiments, Scheffe tests indicated that the mean of the sad groups was reliably greater $(p<.01)$ than the means of either the happy or the neutral groups; however, the means of the happy and neutral groups did not reliably differ on the DACL measurement. ${ }^{1}$

\section{Recall Performance for the Mood-Induction Groups}

Means for the correct recall of letters out of a possible $\mathbf{4 2}$ for each mood group were as follows: in Experiment 1, happy, 19.20; neutral, 27.93; and sad, 19.13 $[F(2,42)=10.57, p<.001]$; in Experiment 2, happy, 16.40; neutral, 25.87; and sad, $17.67[F(2,42)=9.28$, $p<.001]$. For both experiments, Scheffé tests indicated that the mean recall for the neutral groups was reliably greater $(p<.01)$ than it was for either the happy or the sad groups; however, the recall means for the happy and sad groups did not reliably differ from each other.

\section{Number of Total Thoughts Listed According to Mood-Induction Group}

The production of total thoughts listed was similar for all mood-induction groups, as is shown by the following means: Experiment 1, happy, 9.93; neutral, 9.27; and sad, $8.13[F(2,42)=.80, p>.05]$; Experiment 2, happy, 13.27; neutral, 10.93; and sad, $13.13[F(2,42)=$ $.47, p>.05]$.

\section{Proportion of Irrelevant Thoughts Listed According to Mood-Induction Group}

An unweighted proportion of irrelevant thoughts was obtained by simply dividing the number of thoughts that the subjects rated as irrelevant to successful task solution by the total number of thoughts listed. The mean unweighted proportions were as follows: Experiment 1, happy, .62; neutral, . 33 ; and sad, .62 $[F(2,42)=7.49$, $p<.01$ ]; Experiment 2, happy, .56; neutral, .41; and sad, . $55[F(2,42)=2.54, p>.05]$. For Experiment 1 , a Scheffé test revealed that the neutral group reported a reliably lower proportion of irrelevant thoughts than did either the happy or the sad group, both of which were very similar in the number of irrelevant thoughts reported.

As previously noted, a measure that accounts for both judged frequency and judged intensity of irrelevant thoughts provides increased sensitivity over a simple, unweighted proportional measure of irrelevant thoughts. Thus, a single score for each thought was computed that accounted for both judged intensity and judged frequency of the particular thought. This was achieved by arbitrarily multiplying each thought's frequency rating by its intensity rating, yielding a weighted score for each thought. For example, if a single thought was rated as occurring
3 times and its intensity rated as a strong effect (a 4 on the scale), the thought was given a weighted score of 12 . All the weighted scores for each subject were then summed in accordance with whether they were judged as relevant or irrelevant. The sum of the irrelevant weighted scores was divided by the total of all of the weighted scores. This resulted in a weighted irrelevant processing score expressed as a proportion. The mean weighted proportions were the following: in Experiment 1, happy, .51 ; neutral, .16; and sad, . $48[F(2,42)=6.47, p<.01]$; in Experiment 2, happy, .51; neutral, .26; and sad, .40 $[F(2,42)=4.16, p<.05]$. Scheffé tests indicated that the neutral groups in both experiments reported a reliably lower proportion of irrelevant thoughts than did either the happy or the sad groups, which did not differ.

\section{Irrelevant Thoughts, Emotional Mood States, and Cognitive Task Performance}

The relationship between the number of letters correctly recalled and the weighted proportion of irrelevant thoughts are displayed in Figure 1 for Experiments 1 and 2. The means obtained by each group are noted, and a line is provided corresponding to a visual fit. This figure also portrays the negative correlation that was obtained by including all of the subjects in each of the three mood groups [for Experiment 1, $r(44)=-.72, p<.001$; for Experiment $2, r(44)=-.67, p<.001]$. In other words, decreases in recall were shown to be reliably associated with increases in the proportion of irrelevant thoughts for all subjects.

Correlations for the correct number of letters recalled and the weighted proportion of irrelevant thoughts were also computed for each mood group. The within-group correlations were as follows: for Experiment 1, happy, $r(14)=-.69, p<.01$; neutral, $r(14)=-.69, p<.01$; and sad, $r(14)=-.58, p<.05$; for Experiment 2, happy, $r(14)=-.58, p<.05$; neutral, $r(14)=-.64$, $p<.01$; and sad, $r(14)=-.66, p<.01$.

In addition, an overall correlation for the correct number of letters recalled and the unweighted proportion of irrelevant thoughts was computed for all three mood groups combined: for Experiment 1, $r(44)=-.73, p<$ .001 ; for Experiment 2, $r(44)=-.66, p<.001$. Correlations for the correct number of letters recalled and the unweighted proportion of irrelevant thoughts were also computed for each mood group. The within-group correlations were as follows: for Experiment 1, happy, $r(14)$ $=-.65, p<.01 ;$ neutral, $r(14)=-.75, p<.001$; and sad, $r(14)=-.54, p<.05$; for Experiment 2, happy, $r(14)=-.48, p<.05$; neutral, $r(14)=-.73, p<$ .001 ; and sad, $r(14)=-.59, p<.01$.

\section{Multiple Regression Analysis}

The relationship shared by irrelevant thoughts, emotional mood states, and cognitive task performance was investigated via multiple regression. A hierarchical approach was used, with the weighted proportion of irrelevant thoughts entered first and mood-induction condition 


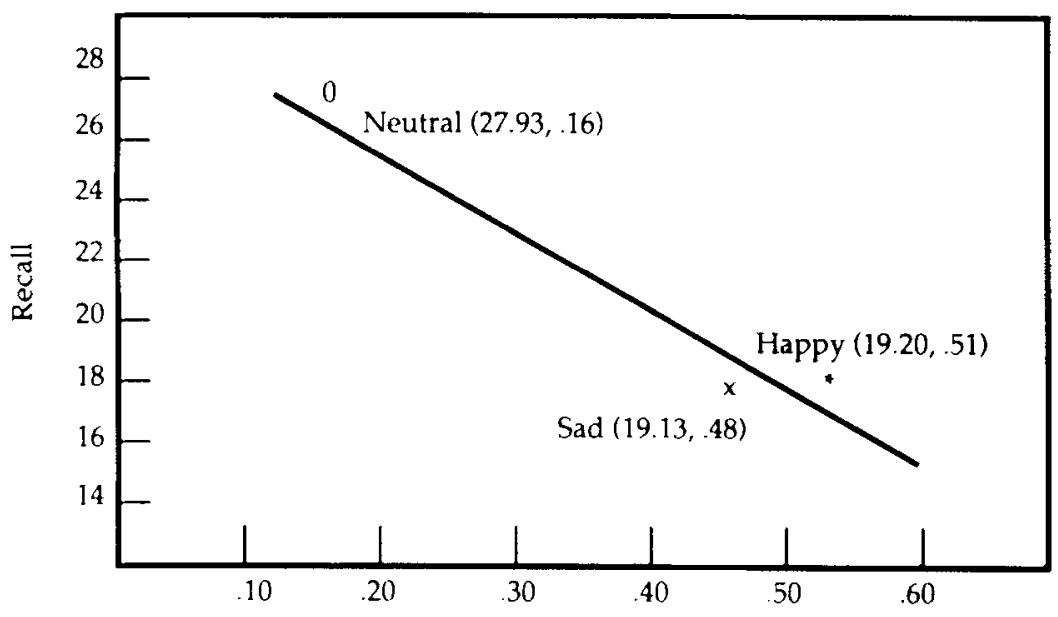

Weighted Proportion of Irrelevant Thoughts

Experiment 1 (delayed)

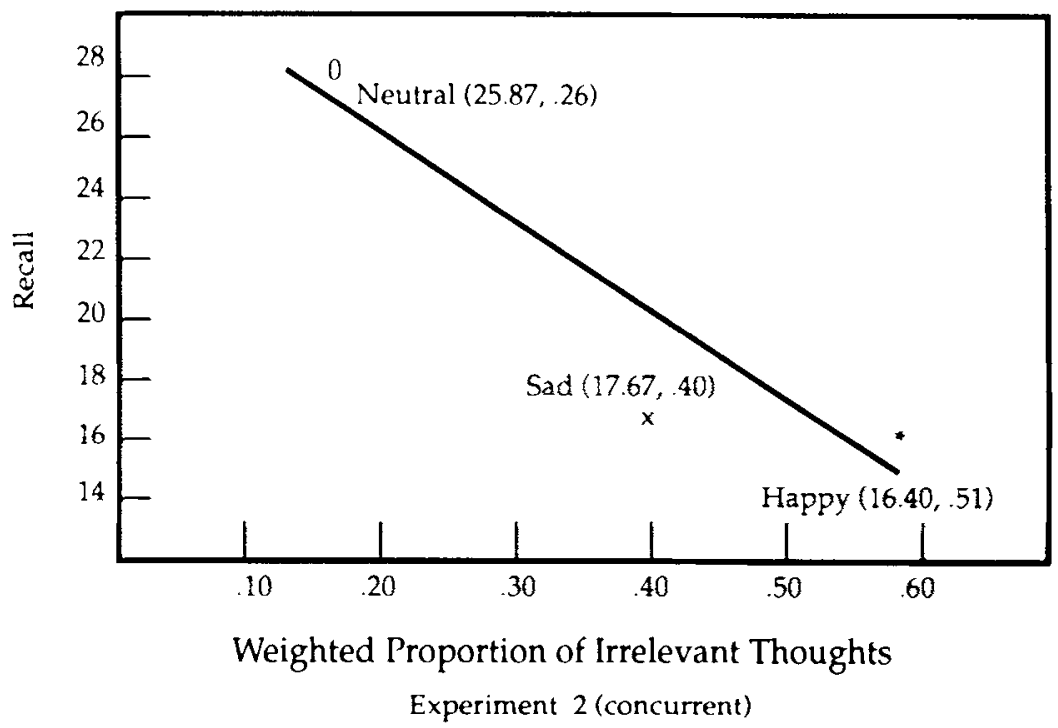

Figure 1. Thought-listing, relationship shared by recall, and weighted proportion of irrelevant thoughts for all groups combined. In Experiment 1 (delayed), $r=-.72$; in Experiment 2 (concurrent), $r=-.67$.

entered second. The proportion of irrelevant thoughts was reliably and negatively related to recall performance, sharing $52 \%$ of the variance in Experiment $1[F(1,39)=$ $53.76, p<.001]$ and $45 \%$ of the variance in Experiment $2[F(1,39)=41.72, p<.001]$.

Emotional mood state was also reliably related to recall performance, sharing $7 \%$ of the variance when the effects of irrelevant thoughts were removed in Experiment 1 $[F(2,39)=3.54, p<.05]$ and $11 \%$ of the variance when the effects of irrelevant thoughts were removed in Experiment $2[F(2,39)=4.83, p<.05]$. Scheffé tests in both experiments showed that the neutral groups recalled reliably more than either the happy or the sad groups, which did not differ from each other.
The proportion of irrelevant thoughts and the moodinduction condition did not significantly interact in their relationship with recall performance in either experiment. Thus, the slope of the relationship between the proportion of irrelevant thoughts and the recall performance was similar for all three groups.

\section{DISCUSSION}

This research produced three primary findings that provide for a greater understanding of the complex cognition/emotion relationship. First, happy and sad subjects reported greater proportions of irrelevant thoughts and performed the memory task more poorly than did sub- 
jects in the neutral (control) group. This finding is consistent with previous research, in which the detrimental effects of emotion-related thoughts have been demonstrated (e.g., Ingram et al., 1987; Ottaviani \& Beck, 1988; Sarason et al., 1986; Seibert \& Ellis, 1991b). As stated earlier, emotional states are accompanied by related, self-referent distracting thoughts. Such thoughts distract focus from the criterion task, so it is not surprising that performance suffers during emotional states.

Most of the research regarding cognitive impairment during emotional mood states has been focused on depression and anxiety. Our second primary finding adds to this research by demonstrating that happy mood states are also associated with decreases in recall and increases in irrelevant thoughts; in fact, the pattern of data obtained from the happy and sad groups is exceptionally similar. This finding supports our thesis that emotional states in general-not just negative states-include thoughts that can impair performance. Anecdotal accounts of the effects of positive states often include statements such as, "I was so happy about our party that I couldn't concentrate on my work" or "I was so eager to leave on our vacation that I forgot to pack the camera." Still, people claim to perform better in happy moods (a point to be discussed later). Our research does not support this claim. We believe that any emotional state includes potential cognitive impairment.

Third, although the proportion of irrelevant thoughts was greater for happy and sad subjects, all subjects reported irrelevant thoughts. Increases in irrelevant thoughts were negatively related to decreases in successful performance of the cognitive task. We found this relationship across and within the happy, sad, and neutral (control) groups. Specifically, for all subjects, the more the irrelevant thoughts reported, the fewer the correct letter sequences recalled.

This finding is consistent with models of attention in which it is suggested that when attention is diverted from the criterion task, performance is likely to suffer. Measurable performance decrements are found when the amount of attentional resources diverted to off-task processing is substantial, leaving inadequate resources to perform the criterion task (Ellis \& Ashbrook, 1988; Hasher \& Zacks, 1979; Kahneman, 1973). Many factors have the potential of evoking irrelevant and distracting thoughts; one such factor is clearly an emotional mood state.

In the context of criticism raised about specific thoughtlisting procedures, the similarities in findings across the two experiments were particularly striking. In each procedure, verbal reports are employed as data; this method is consequently vulnerable to the routine criticisms leveled at the use of such data. Unfortunately, current methodology is such that it is impossible to capture every thought produced. People may forget thoughts, or perhaps be unwilling to express a particular thought. Thoughts may occur that are not expressible because people may not be consciously aware of the particular thought, or a thought may be inexpressible because it is not of a representa- tional form that permits verbal or written expression. Thus, any thought-listing procedure provides at best a sampling of thoughts that do occur. Nevertheless, both procedures yielded the same pattern of results. Hence, the obtained relationship among irrelevant thoughts, emotional mood states, and cognitive task performance appears to be robust.

Another issue concerning the use of verbal protocols involves demand characteristics. The subjects in this and in previous research (see Seibert \& Ellis, 1991b) listed thoughts that indicated they were unaware of the experimental hypothesis. For example, individuals in the happy-mood induction groups consistently reported that they performed well-that a happy mood facilitated their performance. Yet the happy and sad groups performed the recall task similarly. In addition, the happy and sad groups consistently reported similar proportions of irrelevant thoughts. Clearly, the experimental hypothesis was not obvious to the subjects. Conversely, the sad-mood groups reported thoughts conveying perceptions of poor performance, and their performance was poor.

These types of thoughts are consistent with the "sadder but wiser" hypothesis posed by Alloy and Abramson (1979), which suggests that individuals in sad emotional mood states appear to be better able to judge personal behavioral contingencies than do individuals in happy or neutral emotional mood states, who overestimate personal behavioral contingencies. In other words, sad individuals appeared to have a more realistic viewpoint and are thus sadder but wiser.

In summary, people produce more irrelevant thoughts during emotional mood states. When the proportion of irrelevant thoughts to relevant thoughts increases, performance suffers. Thoughts play a dramatic role in the cognition/emotion relationship. Thoughts vary in accordance with emotional states and can inspire or impair cognitive performance. The dynamics of this role must be sufficiently identified to enable more inspiration and less impairment.

\section{REFERENCES}

Alloy, L. B., Abramson, L. Y. (1979). Judgment of contingency in depressed and nondepressed subjects: Sadder but wiser? Joumal of Experimental Psychology: General, 108, 441-485.

Anooshian, L. J., Siegel, A. W. (1985). From cognitive to procedural mapping. In C. J. Brainerd \& M. Pressley (Eds.), Basic processes in memory development: Progress in cognitive development research (pp. 47-101). New York: Springer.

Beck, A. T. (1978). Depression inventory. Philadelphia: Center for Cognitive Therapy.

BOWER, G. H., \& MAYER, J. D. (1989). In search of mood-dependent memory: Theory, research, and applications. Joumal of Social Behavior \& Personality, 4, 121-156.

Bower, G. H., Winzenz, D. (1969). Group structure, coding, and memory for digit series. Journal of Experimental Psychology Monographs, 80(2, Pt. 2), 1-17.

Clark, M. S., Mrlberg, S., \& Erber, R. (1988). Arousal-statedependent memory: Evidence and implications for understanding social judgments and social behavior. In $\mathbf{K}$. Fiedler \& J. Forgas (Eds.), Affect, cognition, and social behavior (pp. 63-83). Toronto: Hogrefe. 
ElLIS, H. C. (1985). On the importance of mood intensity and encoding demands in memory: Commentary on Hasher, Zacks, Rose, Sanft, and Doren. Journal of Experimental Psychology: General, 114, 392-395.

Elus, H. C., \& Ashrrook, P. W. (1988), Resource allocation model of the effects of depressed mood states on memory. In K. Fiedler \& J. Forgas (Eds.), Affect, cognition, and social behavior: New evidence and integrative attempts (pp. 25-43). Toronto: Hogrefe.

Ellus, H. C., Ashbrook, P. W. (1989). The "state" of mood and memory research: A selective review. Journal of Social Behavior \& Personality, 4 (2), 1-21.

Ellis, H. C., Parente, F. J., \& Walker. C. W. (1974). Coding and varied input versus repetition in human memory. Joumal of Experimental Psychology, 102, 284-290.

Ellis, H. C., Seibert, P. S., Herbert, B. J. (1990). Mood state effects on thought listing. Bulletin of the Psychonomic Socierv. 28. 147-150

Folkman, S., Lazarus, R. S. (1985). If it changes it must be a process: Study of emotion and coping during three stages of a college examination. Journal of Personality \& Social Psychology, 48, $150-170$

Forgas, J., Bower, G. H. (1988). Affect in social and personal judgments. In K. Fiedler \& J. Forgas (Eds.), Affect, cognition, and social behavior: New evidence and integrative attempts (pp. 183-208). Toronto: Hogrefe

HASHER, L., ZACKs, R. T (1979). Automatic and effortful processes in memory. Journal of Experimental Psychology: General. 108. 356-388.

Heimberg, R. G., Nyman, D., OBrien, G. T. (1987). Assessing variations of the thought-listing technique: Effects of instructions, stimulus intensity, stimulus modality, and scoring procedures. Cognitive Therapy \& Research, 11, 13-24.

ingram, R. E., Kendall, P. C., Smith, T. W., Donnell, C., RoNAN, K. (1987). Cognitive specificity in emotional distress. Journal of Personality \& Social Psychology, 53, 734-742.

ISEN, A. M. (1984). Toward understanding the role of affect in cognition. In R. S. Wyer \& T. K. Srull (Eds.), Handbook of social cognition (Vol. 3, pp. 179-236). Hillsdale. NJ: Erlbaum.

Kahneman, D. (1973). Attention and effort. Englewood Cliffs, NJ: Prentice-Hall.
Kenfaly, P. M. (1986). The Velten mood induction procedure: A methodological review. Motivation \& Emotion, 10, 315-335.

LeIGHT, K. A., Elus, H. C. (1981). Emotional mood states, strategies, and state-dependency in memory. Journal of Verbal Learning \& Verbal Behavior, 20, 251-266.

LuBIN, B. (1965). Adjective checklists for measurement of depression. Archives of General Psychiatry, 12, 57.62.

Ottaviani, R., \& BeCK. A. (1988). Cognitive theory of depression. In $\mathrm{K}$. Fiedler \& J. Forgas (Eds.), Affect, cognition, and social behavior: New evidence and integrative antempts (pp. 209-218). Toronto: Hogrefe.

SARASON, I. G. (1984). Stress, anxiery, and cognitive interference: Reactions to tests. Joumal of Personality \& Social Psychology, 46, 929-938.

Sarason, I. G.. Sarason, B. R., Keefe, D. E., hayes, B. E., a SheaRIN. E. N. (1986). Cognitive interference: Situational determinants and trait-like characteristics. Joumal of Personalify \& Social Psychology, 51, 215-226.

SARASON, I. G.. STOOPS, R. (1978). Test anxiety and the passage of time. Journal of Consuling and Clinical Psychology, 46, 102-109.

Seibert, P. S., ElLis, H. C. (1991a). A convenient self-referencing mood induction procedure. Bulletin of the Psychonomic Society, 29. 121-124.

SEIBERT, P. S.. \& Elus. H. C. (1991b). Irrelevant thoughts and memory during emotional mood states. Manuscript submitted for publication.

UCRos, C. G. (1989). Mood state-dependent memory: A meta analysis. Cognition \& Emotion, 3, 139-167.

\section{NOTE}

1. Subjects in the happy and neutral groups did not reliably differ on the DACL manipulation check. We used the DACL to be consistent with previous research. However, furure studies that include positive affect should probably be carried out with a test designed to measure the existence, rather than the absence, of positive affect. Such an approach would obviate the potential for floor effects that occurs when one uses the DACL to compare happy and neutral states.

(Manuscript received September 21, 1989; revison accepted for publication March 8, 1991.)

\title{
Notices and Announcements
}

\author{
"New Directions in Portfolio Assessment" \\ Fourth Miami University Conference on the Teaching of Writing \\ Miami University, Oxford, Ohio \\ October 2-4, 1992
}

\begin{abstract}
"New Directions in Portfolio Assessment," the Fourth Miami University Conference on the Teaching of Writing, will be held October 2-4, 1992, at Miami University in Oxford, Ohio.

Proposals are invited on all topics related to college portfolio assessment, including its impact on secondary education. Proposals that focus on research, theory, pedagogy, or any combination of the three are welcome, as are responses to the work of the keynote speakers-Peter Elbow and Edward M. White. Selected papers will be published by Heinemann-Boynton/Cook as a volume of conference essays. Proposal deadline is April 1, 1992.
\end{abstract}

To receive more information or the call for papers, contact Donald A. Daiker, Department of English, Miami University, Oxford, OH 45056 (phone: 513-529-7110 or 5221). 